\title{
High power highly stable passively Q-switched fiber laser based on monolayer graphene
}

\author{
Hanshuo Wu ${ }^{1}$, Jiaxin Song ${ }^{1}$, Jian $\mathrm{Wu}^{1,2, *}$, Jiangming $\mathrm{Xu}^{1,2}$, Hu Xiao ${ }^{1,2}$, Jinyong \\ Leng $^{1,2}$, Pu Zhou ${ }^{1,2,3}$ \\ ${ }^{1}$ College of Optoelectronic Science and Engineering, National University of Defense \\ Technology, Changsha 410073, China \\ ${ }^{2}$ Hunan Provincial Collaborative Innovation Center of High Power Fiber Laser, \\ Changsha 410073, China \\ 33houpu203@163.com \\ *Email:wujian15203@163.com
}

\begin{abstract}
We demonstrate a monolayer graphene based passively Q-switched fiber laser with three-stage amplifiers that can deliver over $80 \mathrm{~W}$ average power at $1064 \mathrm{~nm}$. The highest average power achieved is $84.1 \mathrm{~W}$, with pulse energy of $1.67 \mathrm{~mJ}$. To the best of our knowledge, this is the first time for a high power passively Q-switched fiber laser in the $1 \mu \mathrm{m}$ range reported so far. More importantly, the Qswitched fiber laser operates stably during a week few-hours-per-a-day tests, which proves the stability and practical application value of graphene in high power pulsed fiber lasers.
\end{abstract}

Keywords: fiber laser, Q-switched, high power, highly stable

\section{Introduction}

High power Q-switched fiber lasers are in great demand in material processing, medicine and fundamental research [1-4]. A key approach to generate high average powers is to adopt the master oscillator power amplifier (MOPA) architecture, which usually consists of a seed laser and several stages of power amplifiers. The seed laser provides low power laser emission with stable and low noise output, while the power amplifiers provide power amplification. Up to now, the most powerful pulsed fiber lasers have employed MOPA setup [5-16]. There are some reports on actively Q-switched fiber lasers exploiting MOPA architecture. In the $1 \mu \mathrm{m}$ range, a Q-switched fiber laser with $62 \mathrm{~W}$ average power and $6.2 \mathrm{~mJ}$ pulse energy was reported in 2012[15]; another Q-switched fiber laser with $121 \mathrm{~W}$ average power and $2.4 \mathrm{~mJ}$ pulse energy was presented in 2013[16]; a Q-switched fiber laser with pulse energy of $11 \mathrm{~mJ}$ [9], and a Q- 
switched fiber laser with $230 \mathrm{~W}$ average output power[12] were demonstrated in 2013, and another $300 \mathrm{~W}$ average power Q-switched fiber laser in 2014[13]. In the $2 \mu \mathrm{m}$ range, a singlefrequency actively Q-switched fiber laser with $4.4 \mathrm{~W}$ average output power and $220 \mu \mathrm{J}$ pulse energy was proposed in 2011[11]; a nanosecond Q-switched fiber laser with $50 \mathrm{~W}$ average output, $\sim 1 \mathrm{~mJ}$ pulse energy and $\sim 1 \mathrm{~kW}$ peak power was reported in 2014[14]; and a Q-switched fiber laser that could deliver $110 \mathrm{~W}$ average power was fabricated in 2015[10].

Compared with actively Q-switched fiber lasers, passively Q-switched ones, which usually adopt saturable absorbers [17-24], have the advantages of compactness, simple setup and low cost. However, although passively Q-switched fiber lasers have been intensively studied in recent years, there are few reports about high power passively Q-switched fiber lasers except a Q-switched Tm-doped fiber laser based on graphene oxide (GO) presented by Junqing Zhao et al., which delivers $\sim 73 \mathrm{~W}$ average power at $1950 \mathrm{~nm}$ [1]. Although ytterbium-doped fiber amplifiers (YDFAs) have been proven to be capable of providing high single-pass gain, very high optical-to-optical efficiencies in pulsed laser system, no high power passively Qswitched fiber laser in the $1 \mu \mathrm{m}$ has ever been reported so far.

In this paper, we build a passively Q-switched high power ytterbium-doped fiber laser based on monolayer graphene that can deliver $84.1 \mathrm{~W}$ average power. The pulse operation and stability of the seed laser as well as the performance of the amplifiers are investigated in detail. To the best of our knowledge, this is the first time for a high power passively Q-switched fiber laser reported in the $1 \mu \mathrm{m}$ range.

\section{Material Characterization and Experimental Setup}

\subsection{Material Characterization}

(a)

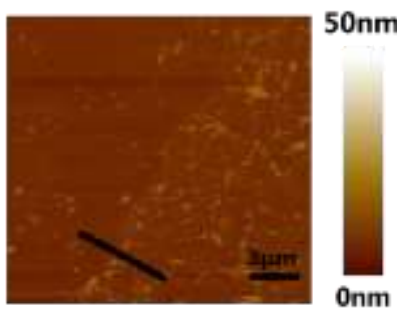

(c)

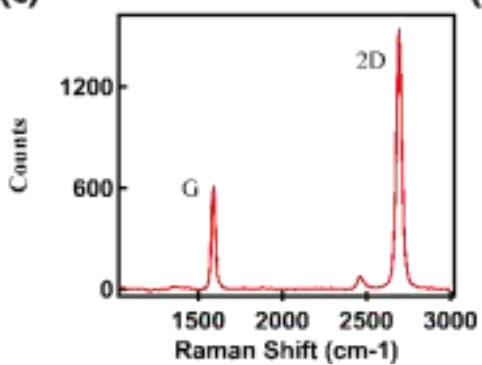

(b)

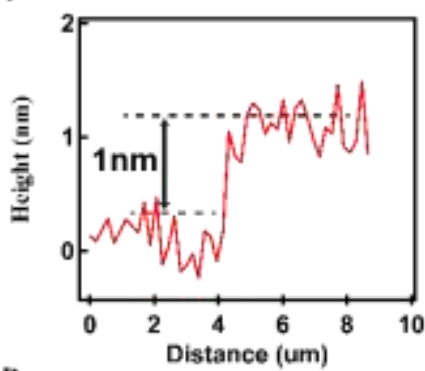

(d)

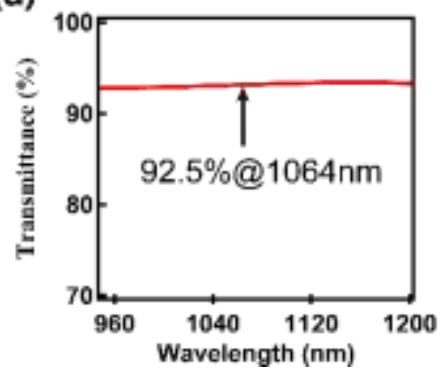

Figure 1. Material characterization: (a) AFM image and (b) surface profile of graphene sample (c) Raman shift and (d) transmittance spectrum of graphene sample 
The monolayer graphene adopted in our work is bonded on the end face of a fiber patch cord, which is prepared by a similar approach as previous works [25]. The graphene sample is characterized by an atomic force microscopy (AFM), with which the roughness and thickness of sample is measured at the size of $15 \mu \mathrm{m} \times 15 \mu \mathrm{m}$. The AFM image is shown in Figure 1(a). The surface profile along the black line in Figure 1(a) is plotted in Figure 1(b) and the height difference between the graphene sample and the substrate is about $1 \mathrm{~nm}$, which means the graphene sample is single layer.

Raman scattering measurements are carried out using a commercial confocal microscope Raman system, excited by $473 \mathrm{~nm}$ laser. A 50x objective is used for both laser excitation and Raman scattering collection. Figure 1(c) is the Raman shift spectrum of the sample. The prominent $\mathrm{G}$ and 2D peaks are located at $1593.3 \mathrm{~cm}^{-1}$ and $2693.7 \mathrm{~cm}^{-1}$, and the corresponding full width half magnitude (FWHM) are 24 and $37 \mathrm{~cm}^{-1}$, respectively. The ratio of G/2D is about 0.39 . Thus, the AFM data and Raman spectroscopy can clearly distinguish the monolayer graphene.

At the meantime, the broadband spectrum transmission property of the graphene samples is characterized. A self-constructed supercontinuum source emitting from $\sim 600 \mathrm{~nm}$ to $\sim 2000$ $\mathrm{nm}$ is used to measure the transmission spectrum. As is shown in Figure 1(d), the transmittance is around $92 \% \sim 93 \%$ from $950 \mathrm{~nm}$ to $1200 \mathrm{~nm}$ and the transmittance at our working wavelength (i.e. $1064 \mathrm{~nm}$ ) is about $92.5 \%$.

\subsection{Experimental Setup}

The MOPA-based passively Q-switched fiber laser system configuration is depicted in Fig 2, which is comprised of a seed laser followed by multi-stage amplifiers.

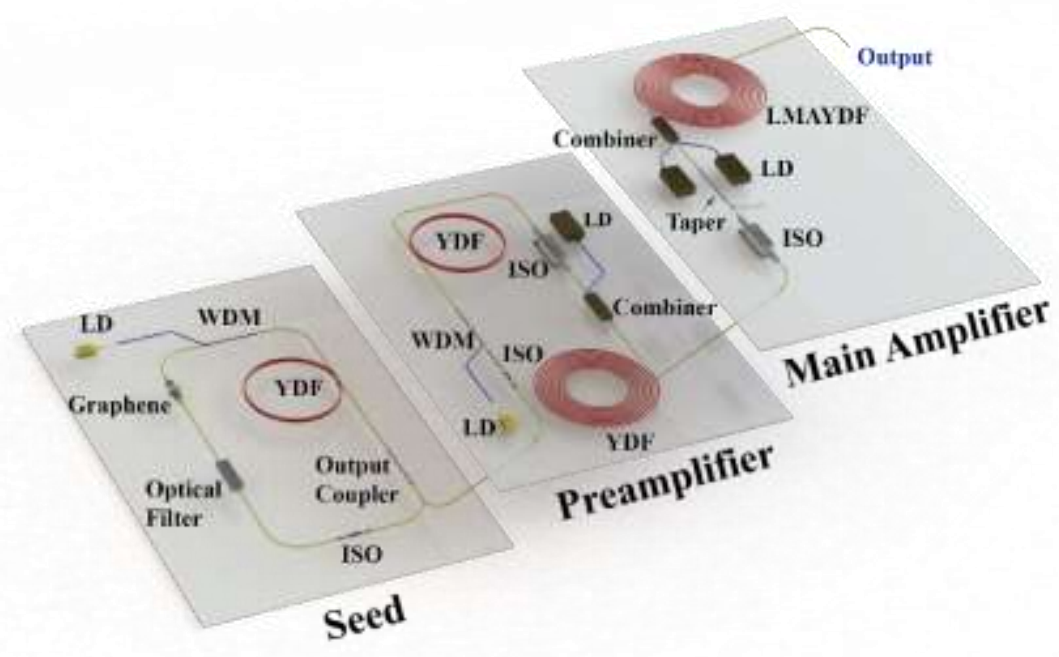

Figure 2. Experimental setup of the passively Q-switched fiber laser with 3-stage amplifiers

The seed laser consists of the following components: a single mode laser diode (LD) centered at $976 \mathrm{~nm}$ to provide pump power, a wavelength division multiplexer (WDM, $976 / 1064 \mathrm{~nm}$ ) to couple the pump light into the ring cavity, a piece of 2.5 meters long ytterbiumdoped fiber (YDF, 6/125 $\mu \mathrm{m}, \mathrm{NA}=0.22$ ) as gain medium, whose absorption is $12 \mathrm{~dB} / \mathrm{m}$ at 976 
$\mathrm{nm}$, an optical filter centered at $1064 \mathrm{~nm}$ to select the oscillation wavelength, a 30/70 coupler as output coupler, an isolator (ISO) to ensure unidirectional propagation, and the monolayer graphene sample to serve as saturable absorber. The total cavity length is $\sim 10 \mathrm{~m}$.

The Q-switched pulses are then delivered into the amplifiers. An ISO is inserted between the seed laser and the amplifiers to avoid any reflected light that may induce any instability or damage.

The preamplifier includes two-stage amplifiers. The first-stage amplifier uses a 976/1064 $\mathrm{nm}$ WDM to launch the $976 \mathrm{~nm}$ pump light into a piece of 2.5 meters long YDF, which is the same as the gain fiber used in the seed laser. The second stage amplifier adopts a piece of 3 meters long double-clad YDF $(10 / 125 \mu \mathrm{m}$, core NA=0.09) whose cladding absorption coefficient is $\sim 6 \mathrm{~dB} / \mathrm{m}$ at $976 \mathrm{~nm}$, and a $10 \mathrm{~W}$ multimode $\mathrm{LD}$ centered at $976 \mathrm{~nm}$ is used as pump source. The pump light is coupled into the active fiber through a $(1+1) \times 1$ combiner. The third stage, i.e. the main amplifier, uses a piece of 3 meters long large mode area (LMA) doubleclad YDF $(20 / 130 \mu \mathrm{m}$, core $\mathrm{NA}=0.09)$, the cladding absorption of which is $\sim 3.1 \mathrm{~dB} / \mathrm{m}$ at 915 $\mathrm{nm}$, pumped by two $50 \mathrm{~W}$ multimode LDs centered at $976 \mathrm{~nm}$ via a $(2+1) \times 1$ combiner. There is an isolator between each stage amplifier to block possible counter-propagating light from the subsequent amplifier. A 99/1 taper is inserted before the main amplifier to monitor the backward light in case strong stimulated Raman scattering (SRS) occurs. The LD used in the second- and third-stage as well as the LMA YDF are water-cooled to $20{ }^{\circ} \mathrm{C}$ to avoid thermal damage.

\section{Results and discussions}

Based on the above-mentioned experimental setup, the overall properties of the passively Qswitched fiber laser with MOPA configuration are investigated. The pulses are measured and recorded by a $5 \mathrm{GHz}$ photodetector together with a $2 \mathrm{GHz}$ oscilloscope. An optical spectrum analyzer (OSA) is used to monitor the output spectrum of the laser system.

\subsection{Performance of the seed laser}


(a)

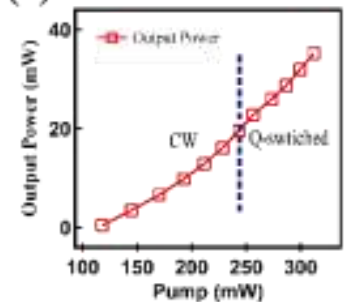

(c)

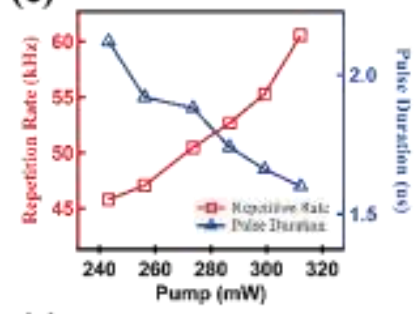

(e)

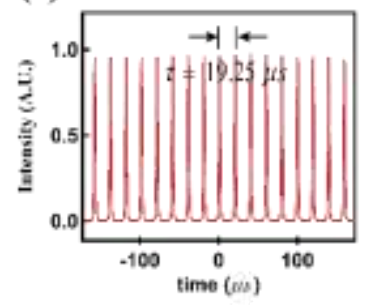

(b)

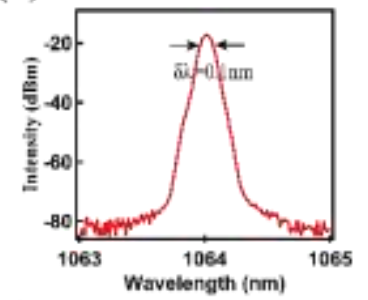

(d)

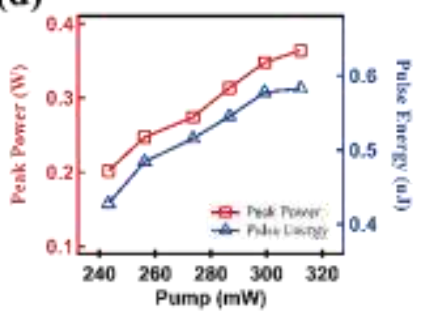

(f)

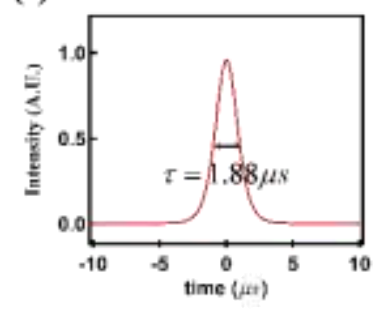

Figure 3. Performance of the seed laser: (a) output power against pump power (b) emission spectrum (c) repetition rate and pulse duration and (d) pulse energy and peak power as a function of pump power (e) pulse train and (d) profile of a single pulse at the pump power of $273 \mathrm{~mW}$.

The laser system achieves self-starting Q-switched operation by increasing the pump power over a certain value. Fig 3(a) presents the variation of the output power of the seed laser as a function of the pump power. It starts to lase at the pump power of $118 \mathrm{~mW}$, and goes into Qswitched regime when the pump power exceeds $243.3 \mathrm{~mW}$. The maximum output power reaches $35.3 \mathrm{~mW}$ at the pump of $312.2 \mathrm{~mW}$. The overall efficiency is relatively low mainly owing to the insertion loss brought by the graphene and optical filter. Fig 3(b) is the output spectrum of the laser. Since the spectrum is mainly determined by the optical filter, thus no obvious linewidth broaden is observed while increasing the pump power. The linewidth is $\sim 0.1$ $\mathrm{nm}$ centering at $1064 \mathrm{~nm}$ at the maximum pump power.

We also investigated the evolution of Q-switched pulses as a function of the pump power. Fig 3(c) gives the variation of the pulse repetition rate and the pulse duration against the pump power. The repetition rate varies from $45.79 \mathrm{kHz}$ to $60.54 \mathrm{kHz}$ when the pump power increases from $243.3 \mathrm{~mW}$ to $312.2 \mathrm{~mW}$, while the pulse duration decreases from $2.12 \mu$ s to $1.6 \mu \mathrm{s}$ simultaneously, which is the typical characteristics of Q-switched operation. Higher repetition rate and shorter pulse duration can be expected if higher pump power is provided. Fig 3(d) depicts the pulse peak power and pulse energy varying with the pump power, both of which increase monotonously: the former of which increases from $0.20 \mathrm{~W}$ to $0.36 \mathrm{~W}$, and the latter of which increases from $0.43 \mu \mathrm{J}$ to $0.58 \mu \mathrm{J}$. 
We fix the pump power at $273 \mathrm{~mW}$, where the output power is $26.01 \mathrm{~mW}$, to make further power amplification, the time interval of adjacent pulses and pulse duration of which are 19.25 $\mu$ s and $1.88 \mu$ s, as shown in Fig 3(e) and (f), respectively.

In order to test the stability of our laser system, we conduct a week of few-hours-per-a-day tests of the seed laser, and record the time interval of adjacent pulses, the pulse duration as well as the output power at the pump of $273 \mathrm{~mW}$. The pulse-pulse interval and pulse duration only experience insignificant change ( $\sim .8 \%$ variation of the pulse-pulse interval and $\sim 2 \%$ variation of the pulse duration from the average) during the whole tests, as shown in Fig 4(a), which indicates the pulse operation is very stable and can endure long-term tests. Fig 4(b) shows the variation of output power at the pump of $273 \mathrm{~mW}$ during a week's per-day tests. The variation is slight (less than 3\% variation around the average output power), which further confirms the stability of the seed laser. These results reveal that graphene is an ideal candidate to function as highly stable saturable absorbers in pulsed fiber lasers.

(a)

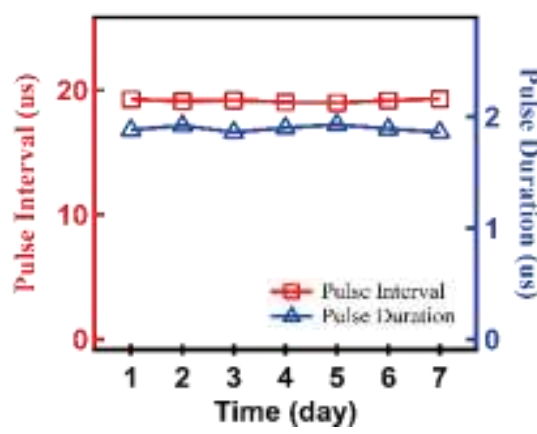

(b)

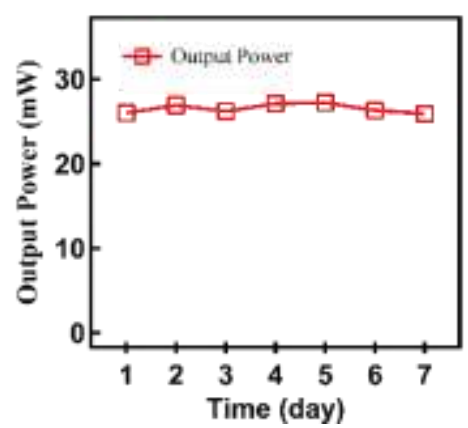

Figure 4. Stability of the seed laser: (a) variation of the pulse-pulse interval and pulse duration (b) stability of the output power

\subsection{Performance of the amplifier}

The preamplifier consists of the first two stage amplifiers. The output power is amplified from $26.01 \mathrm{~mW}$ to $236.9 \mathrm{~mW}$ by the first stage amplifier, after which it is further boosted to $3.95 \mathrm{~W}$ by the second stage amplifier. No amplified spontaneous emission (ASE) or SRS is observed under this situation. Then the output of the preamplifier is delivered into the main amplifier to make further power amplification. 
(a)

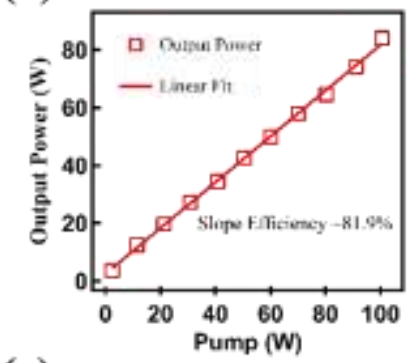

(c)

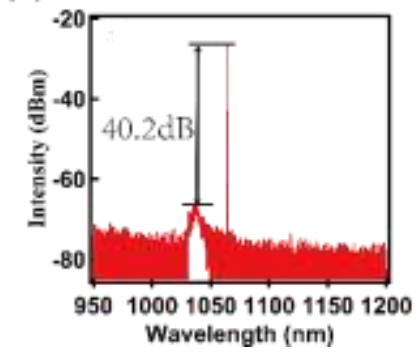

(b)

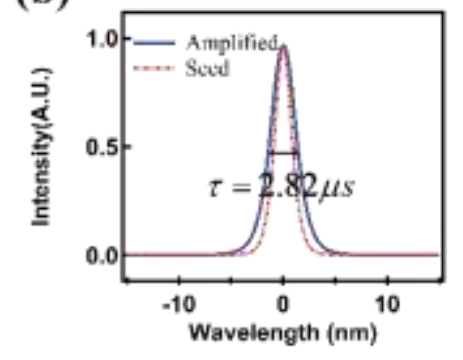

(d)

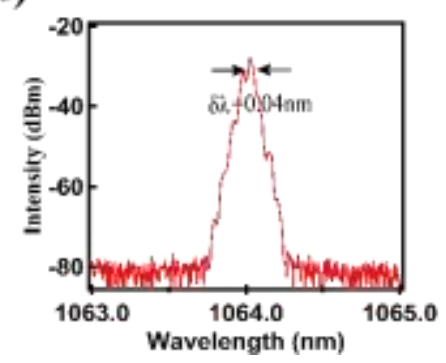

Figure 5. Performance of the main amplifier: (a) output power versus pump power (b) profile of the pulse before and after amplification (c) output spectrum in a large wavelength range and (d) fine output spectrum at the pump power of $100.65 \mathrm{~W}$

The performance of the main amplifier is studied in detail, the results of which are depicted in Fig 5. Fig 5(a) presents the output power against the pump power launched into the main amplifier with linear fit. The output power increases almost linearly with a slope efficiency of $81.9 \%$, with the maximum output power of $84.1 \mathrm{~W}$ at the pump of $100.65 \mathrm{~W}$. The profile of a single pulse at the maximum output versus the pulse of the seed laser are depicted in Fig 5(b). The pulse duration increases to $2.82 \mu \mathrm{s}$, while the repetition rate remains the same, with peak power of $590.85 \mathrm{~W}$ and pulse energy of $1.67 \mathrm{~mJ}$. The spectrum at the maximum pump power spanning from $950 \mathrm{~nm}$ to $1200 \mathrm{~nm}$ is shown in Fig 5(c). The difference value between the signal amplitude and the maximum ASE intensity can reach $40.2 \mathrm{~dB}$ and no parasitic lasing or SRS is observed, which indicates it has potential for further power scaling. The fine spectrum is shown in Fig 5(d), and the linewidth is narrowed to $\sim 0.04 \mathrm{~nm}$.

\section{Conclusion}

In conclusion, we propose and demonstrate a passively Q-switched fiber laser with 84.1 $\mathrm{W}$ average output power emitting at $1064 \mathrm{~nm}$ based on monolayer graphene. The peak power reaches $590.85 \mathrm{~W}$ with pulse energy of $1.67 \mathrm{~mJ}$, and the repetition rate and pulse duration are $50.47 \mathrm{kHz}$ and $2.82 \mu$ s, respectively. The slope efficiency of the main amplifier is about $81.9 \%$, and the ASE suppression is about $40.2 \mathrm{~dB}$. No parasitic lasing or SRS are observed in the MOPA. Our work proves the availability and practicality value of graphene saturable absorber in highly stable, high power pulsed fiber lasers. To the best of our knowledge, this is the first time for a passively Q-switched high power fiber laser reported so far in the $1 \mu \mathrm{m}$ range. The output power is limited by the pump power. Further increase of the output power can be expected by increasing the pump power of the amplifier and adding amplifier stages. 


\section{References:}

[1] Zhao J, Zheng Z, Ouyang D and Liu M 201670 W Graphene-Oxide Passively Q-Switched Thulium-Doped Double-Clad Fiber Laser IEEE J SEL TOP QUANT 231

[2] Williams R J, Jovanovic N, Marshall G D and Withford M J 2010 All-optical, actively Q-switched fiber laser OPT EXPRESS 18 7714-23

[3] Tang Y, Yu X, Li X, Yan Z and Wang Q J 2014 High-power thulium fiber laser Q switched with single-layer graphene. OPT LETT 39 614-7

[4] Huang J Y, Huang S C, Chang H L, Su K W, Chen Y F and Huang K F 2008 Passive Q switching of Er- $\mathrm{Yb}$ fiber laser with semiconductor saturable absorber. OPT EXPRESS 16 3002-7

[5] Klenke A, Hädrich S, Eidam T, Rothhardt J, Kienel M, Demmler S, Gottschall T, Limpert J and Tünnermann A 201422 GW peak-power fiber chirped-pulse-amplification system. OPT LETT 396875

[6] Wan P, Yang L M and Liu J 2013 All fiber-based Yb-doped high energy, high power femtosecond fiber lasers OPT EXPRESS 2129854

[7] Eidam T, Hanf S, Seise E, Andersen T V, Gabler T, Wirth C, Schreiber T, Limpert J and Tünnermann A 2010 Femtosecond fiber CPA system emitting $830 \mathrm{~W}$ average output power. OPT LETT 35 94-6

[8] Peng X, Kim K, Mielke M, Jennings S, Masor G, Stohl D, Chavez-Pirson A, Nguyen D T, Rhonehouse D and Zong J 2014 Monolithic fiber chirped pulse amplification system for millijoule femtosecond pulse generation at $1.55 \mu \mathrm{m}$. OPT EXPRESS 22 2459-64

[9] Fang Q, Qin Y, Wang B and Shi W 201311 mJ all-fiber-based actively Q-switched fiber master oscillator power amplifier LASER PHYS LETT 105103

[10] Ouyang D Q, Zhao J Q, Zheng Z J and Ruan S C 2015110 W All Fiber Actively Q-Switched Thulium-Doped Fiber Laser Photonics Journal IEEE 7 1-6

[11] Shi W, Petersen E B, Nguyen D T, Yao Z, Chavez-Pirson A, Peyghambarian N and Yu J 2011220 $\mu \mathrm{J}$ monolithic single-frequency Q-switched fiber laser at $2 \mu \mathrm{m}$ by using highly Tm-doped germanate fibers. OPT LETT 363575

[12] Fang Q, Qin Y, Wang B and Shi W $2013230 \mathrm{~W}$ average-power all-fiber-based actively Q-switched fiber master oscillator - power amplifier APPL OPTICS $\mathbf{5 2}$ 6744-7

[13] Shi W, Fang Q, Tian X and Fan J 2014 300-W-average-power monolithic actively Q-switched fiber laser at $1064 \mathrm{~nm}$ LASER PHYS 2465102

[14] Tang Y, Li X, Yan Z, Yu X, Zhang Y and Wang Q J 2014 50-W 2- $\mu \mathrm{m}$ Nanosecond All-Fiber-Based Thulium-Doped Fiber Amplifier IEEE J SEL TOP QUANT 20 1-7

[15] Zhang H, Wang X, Zhou P, Gong Z and Xu X 20126 mJ, high-average-power, all-fiberized Qswitched fiber master oscillator power amplifier with low repetition rate. APPL OPTICS 516933

[16] Gong Z, Zhang H, Wang X, Zhou P and Jiang H 2013 A 2.4 mJ all-fiber-based Q-switched fiber master oscillator power amplifier LASER PHYS $\mathbf{2 3} 25102$

[17] Lee J, Koo J, Chi C and Lee J H 2014 All-fiberized, passively Q-switched $1.06 \mu \mathrm{m}$ laser using a bulk-structured $\mathrm{Bi}_{2} \mathrm{Te}_{3}$ topological insulator J OPTICS-UK 1685203

[18] Wang X D, Luo Z C, Liu H, Zhao N, Liu M, Zhu Y F, Xue J P, Luo A P and Xu W C 2015 Gold nanorod as saturable absorber for Q-switched $\mathrm{Yb}$-doped fiber laser OPT COMMUN 346 21-5

[19] Kang Z, Guo X, Jia Z, Xu Y, Liu L, Zhao D, Qin G and Qin W 2013 Gold nanorods as saturable absorbers for all-fiber passively Q-switched erbium-doped fiber laser OPT MATER EXPRESS 3 198691

[20] Wang M, Chen C, Huang C and Chen H 2014 Passively Q-switched Er-doped fiber laser using a 
semiconductor saturable absorber mirror Optik - International Journal for Light and Electron Optics 125 2154-6

[21] He X, Zhang H, Lin W, Wei R, Qiu J, Zhang M and Hu B 2015 PVP-Assisted Solvothermal Synthesis of High-Yielded $\mathrm{Bi}_{2} \mathrm{Te}_{3}$ Hexagonal Nanoplates: Application in Passively Q-Switched Fiber Laser SCI REP-UK 515868

[22] Chen B, Zhang X, Wu K, Wang H, Wang J and Chen J 2015 Q-switched fiber laser based on transition metal dichalcogenides $\mathrm{MoS}_{2}, \mathrm{MoSe}_{2}, \mathrm{WS}_{2}$, and $\mathrm{WSe}_{2}$. OPT EXPRESS 2326723

[23] Popa D, Sun Z, Hasan T, Torrisi F, Wang F and Ferrari A C 2011 Graphene Q-switched, tunable fiber laser APPL PHYS LETT 9873106

[24] Li J, Luo H, He Y, Liu Y, Luo B, Sun Z, Zhang L and Turitsyn S K 2014 Semiconductor saturable absorber mirror passively Q-switched $2.97 \mu \mathrm{m}$ fluoride fiber laser LASER PHYS LETT 1165102

[25] Wu J, Xu Y, Xu P, Pan Z, Chen S, Shen Q, Zhan L, Zhang Y and Ni W 2015 Surface-enhanced Raman scattering from AgNP-graphene-AgNP sandwiched nanostructures NANOSCALE 7 17529-37 